\title{
Wissenstransfer im universitären Kontext
}

\section{Unterschiede und Gemeinsamkeiten zwischen den GSK und anderen wissenschaftlichen Disziplinen}

Markus Hayden

Wissenstransfer zählt als eminenter Aspekt der third mission zu den Kernaufgaben der Universitäten (Trencher et al. 2014). Darüber hinaus bietet der Prozess Vorteile für alle beteiligten Parteien (Caldera and Debande 2010; Lightowler and Knight 2013; Wutti and Hayden 2017). Dennoch kommt dem Thema Wissenstransfer im universitären Alltag nur wenig Anerkennung zu. Da Wissenschafter_innen für einen Fortschritt ihrer Karriere dazu angehalten sind, ihre Leistungen auf den Bereich ,Science to Science' zu fokussieren, bleiben andere Tätigkeiten weitgehend unbeachtet. Entsprechend werden Wissenstransferleistungen häufig nur als freiwillige oder außerordentliche Tätigkeiten gewertet. Die einzigen Ausnahmen stellen in der Regel jene Leistungen dar, die den Bereichen Verwertung bzw. Industrie- und Wirtschaftskooperationen zuzuordnen sind. Diese Schieflage in der Anerkennung von Wissenstransferleistungen spiegelt sich auch in anderen Bereichen des Universitätsalltages wieder. So existiert beispielsweise verhältnismäßig viel Forschung zum Wissenstransfer in ökonomischen und technologischen Kontexten (für Reviews diesbezüglich siehe z.B. Agrawal 2001; Geuna and Muscio 2009; Perkmann et al. 2013), wohingegen andere Bereiche bisher nur unzureichend beforscht wurden (Olmos-Peñuela, Castro-Martínez, and D’Este 2014; 
Wutti and Hayden 2017; Hayden, Petrova, and Wutti 2018). Auch global angewendete Kennzahlen und Benchmarks für Wissenstransfer fokussieren sich für gewöhnlich auf wirtschaftliche Aspekte (Patentierungen, Spin-offs, etc.) und Themen der Kollaboration zwischen Universitäten und Firmen in Wirtschaft und Industrie (European Commission 2013). Sogar internationale Universitätsrankings, wie beispielsweise U-Multirank (www.umultirank.org), inkludieren lediglich wirtschaftlich orientierte Kennzahlen, obwohl deren Aussagekraft vonseiten der Wissenschaft häufig kritisiert wird (Agrawal and Henderson 2002; Cohen, Nelson, and Walsh 2002; Olmos-Peñuela, Benneworth, and Castro-Martinez 2014; Hayden et al. 2018). Vor allem in den GSK-Wissenschaften sind Verbindungen zwischen Universitäten und privatwirtschaftlichen Unternehmen unreliable und invalide Benchmarks, da der Wissenstransfer in diesen Disziplinen deutlich häufiger von der Dissemination von Forschungsergebnissen zu Politik, beruflicher Praxis und Zivilgesellschaft gekennzeichnet ist (siehe Abschnitt 1.1).

Aufgrund der geringen akademischen Wertschätzung für Wissenstransferleistungen, die über Themen von Verwertung und Wirtschaftskollaborationen hinausgehen, ist es fraglich, was Wissenschaftler_innen dazu motiviert, in diesem Bereich aktiv zu werden. Diesbezüglich ist festzuhalten, dass bisher kaum Untersuchungen existieren, die sich mit der Einstellung von Forschenden gegenüber dem Themenkomplex Wissenstransfer beschäftigen (Wutti and Hayden 2017; Molas-Gallart and Castro-Martínez 2007).

\section{Forschungsfragen}

Der quantitative Teil der WTZ-Studie beschäftigte sich einerseits mit der Frage, welche Komponenten und Teildisziplinen explizit mit dem Themenkomplex Wissenstransfer verbunden werden. Zudem analy- 
sierten wir Motivatoren und Hindernisse, die ein Engagement in diesem Bereich beeinflussen. Im letzten Teil der Studie wurden die Befragten gebeten, eine Reihe von Aussagen auf einer 7-Punkt-LikertSkala hinsichtlich der persönlichen Zustimmung zu bewerten. Die Aussagen bezogen sich auf Wahrnehmung, Bedeutung und Stellenwert des Themas Wissenstransfer in der wissenschaftlichen Community sowie in der Gesellschaft. Wie bereits beim qualitativen Teil der Studie wurden auch im quantitativen Teil die Unterschiede zwischen den Geistes-, Sozial- und Kulturwissenschaften (GSKWissenschaften) und anderen wissenschaftlichen Disziplinen analysiert.

\section{Ergebnisse}

\section{Definition}

Wie im vorangegangenen Artikel beschrieben, fragten wir im ersten Teil der Studie nach einer Definition für den Begriff Wissenstransfer sowie nach Stichwörtern, die mit dem Begriff assoziiert werden. Im darauffolgenden Abschnitt präsentierten wir eine Liste potenzieller Teilaspekte. Die Teilnehmer_innen sollten jeden der Aspekte hinsichtlich seines Zusammenhangs zum Themenkomplex Wissenstransfer bewerten - unabhängig davon, ob der jeweilige Aspekt Teil der vorangegangenen Definition war oder nicht. Der Grad der Zustimmung ist in Abbildung 1 dargestellt. Zur Überprüfung der Unterschiede zwischen GSK-Wissenschaftler_innen und Wissen-schaftler_innen anderer Disziplinen wurde für jedes Item ein $t$-Test für unabhängige Stichproben gerechnet. Die signifikanten Ergebnisse sind in Tabelle 1 dargestellt.

Wie in der Tabelle ersichtlich, wurden die Kategorien, Wissenschaftskommunikation und Öffentlichkeitsarbeit, , Vorträge/Tagungen außer- 
halb der Scientific Community', ,Organisation von Ausstellungen', ,Kooperationen mit Vereinen' und ,Beteiligung in außeruniversitären Gremien' signifikant häufiger von GSK-Wissenschaftler_innen als Teilaspekte des Themas Wissenstransfer benannt. Im Gegensatz dazu wurden die Kategorien ,Publikationen für spezifische Berufsgruppen', ,Kooperationen mit Firmen/Konzernen', ,Vermarktung von univ. gewonnenem Wissen' sowie ,Spin-Offs' signifikant häufiger von Wissenschaftler_innen anderer Disziplinen ausgewählt. Die Unterschiede waren jedoch, mit Ausnahme der Kategorie ,Spin-Offs, nur von geringer Effektstärke (Cohen, 1988). 


\section{Motivatoren und Hindernisse}

Im folgenden Abschnitt explorierten wir, welche Faktoren zu einem Engagement im Bereich Wissenstransfer motivieren. Den Teilnehmer_innen wurde hierfür eine Liste mit möglichen Motivatoren vorgelegt. Jedes Item der Liste sollte wie im vorangegangenen Abschnitt bewertet werden. Die Ergebnisse sind in Abbildung 2 dargestellt.

\begin{tabular}{|c|c|c|}
\hline & $\begin{array}{l}\text { Mittelwerts- } \\
\text { unterschied }\end{array}$ & $\begin{array}{l}\text { Effektstärke } \\
\left(d_{\text {Cohen }}\right)\end{array}$ \\
\hline $\begin{array}{l}\text { Wissenschaftskommunikation und } \\
\text { Öffentlichkeitsarbeit }\end{array}$ & $-.100^{* *}$ & .388 \\
\hline $\begin{array}{l}\text { Vorträge/Tagungen außerhalb der Scientific } \\
\text { Community }\end{array}$ & $-.105^{*}$ & .311 \\
\hline Publikationen für spezifische Berufsgruppen & $.141^{*}$ & -.291 \\
\hline Organisation von Ausstellungen & $-.196^{* *}$ & .399 \\
\hline Kooperationen mit Firmen/Konzernen & $.231^{* * *}$ & -.472 \\
\hline Kooperationen mit Vereinen & $-.164^{* *}$ & .332 \\
\hline Beteiligung in außeruniversitären Gremien & $-.203^{* *}$ & .418 \\
\hline Vermarktung von univ. gewonnenem Wissen & $.208^{* *}$ & -.437 \\
\hline Spin-Offs & $.240^{* * *}$ & -.559 \\
\hline \multicolumn{3}{|c|}{$\begin{array}{l}\text { Tabelle 1: Ergebnisse der } t \text {-Tests für unabhängige Stichproben } \\
\text { (nichtsignifikante Ergebnisse sind in der Tabelle nicht angegeben) } \\
{ }^{*}=p<.05 ;{ }^{* *}=p<.01 ;{ }^{* * *}=p<.001\end{array}$} \\
\hline
\end{tabular}


Auch bei diesem Teilbereich wurden $t$-Tests für unabhängige Stichproben angewendet, um mögliche Unterschiede im Antwortverhalten aufzuzeigen. Die signifikanten Ergebnisse sind in Tabelle 2 dargestellt.

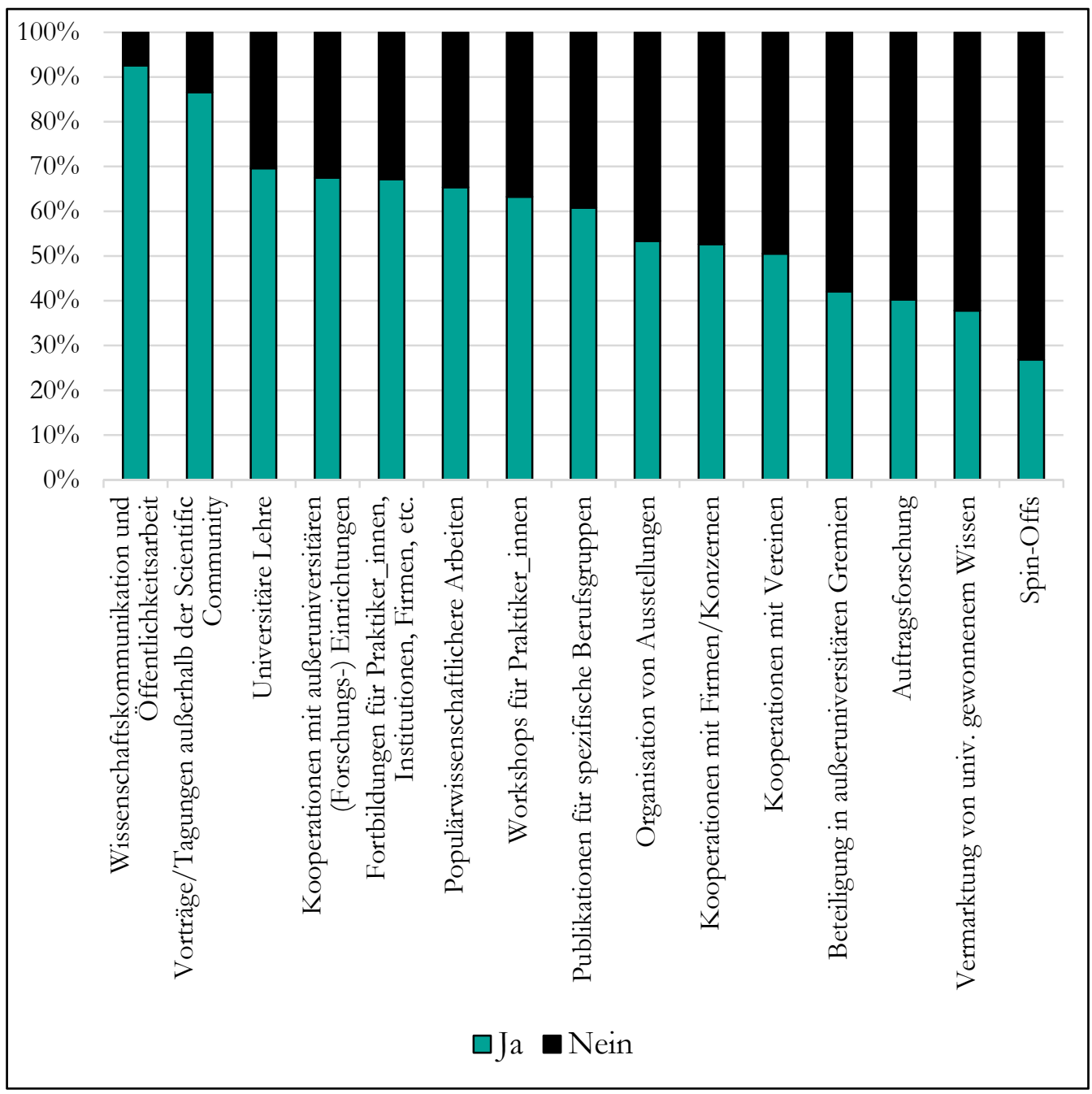

Abbildung 1: Grad der Zustimmung zu unterschiedlichen Aspekten des Themenbereichs Wissenstransfer 


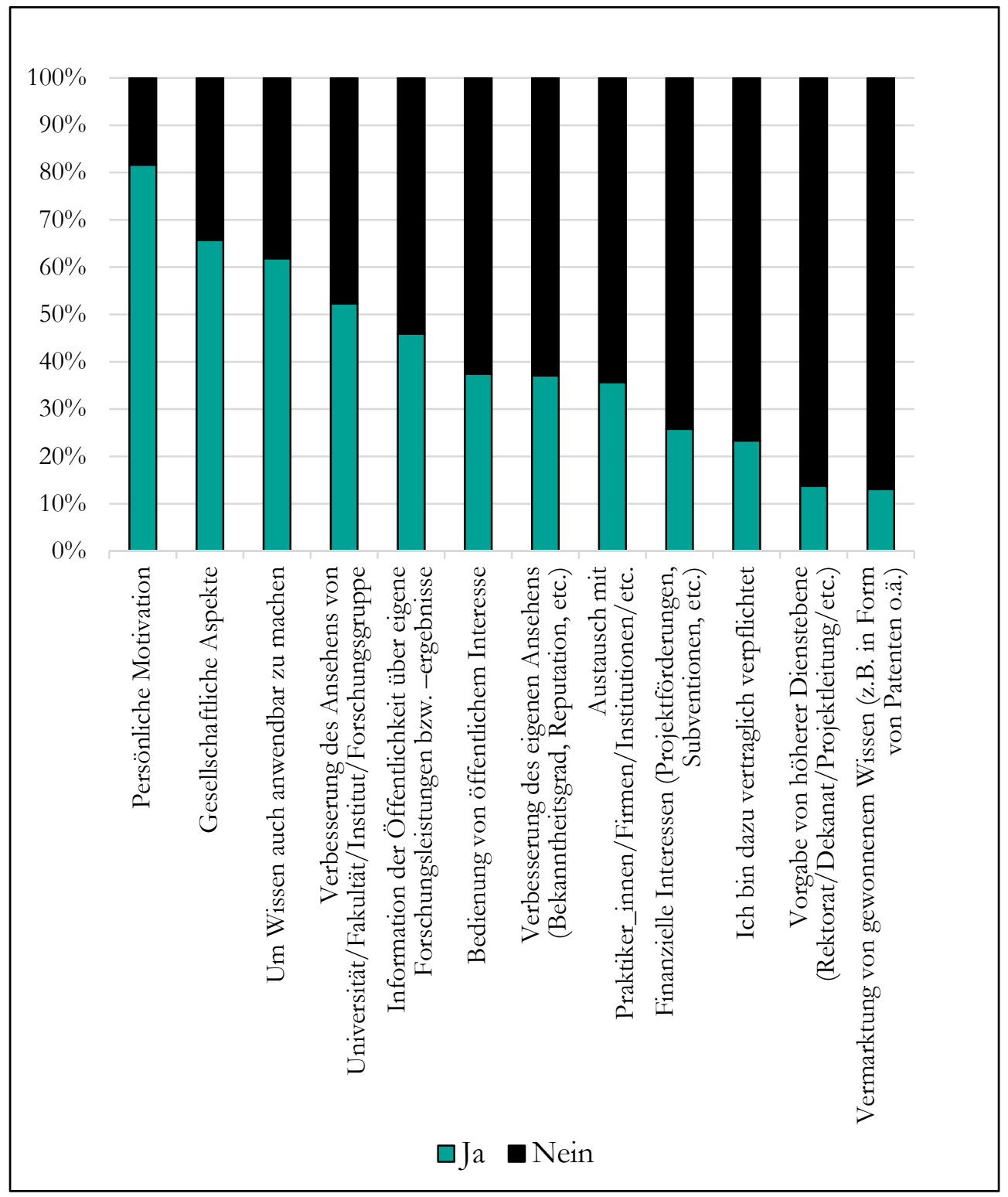

Abbildung 2: Grad der Zustimmung zu unterschiedlichen Motivatoren für Wissenstransfer 
Auch wenn keine großen Unterschiede zwischen den unterschiedlichen wissenschaftlichen Disziplinen festzustellen sind, so ist doch erkennbar, dass Forscher_innen im Bereich der GSK-Wissenschaften stärker durch gesellschaftliche Aspekte zum Wissenstransfer motiviert werden. Im Gegensatz dazu sind in den anderen wissenschaftlichen Disziplinen praxisrelevante und ökonomische Anreize förderlicher. Hierbei ist jedoch zu beachten, dass die meisten Items dieser Kategorien nur von der Minderheit der Teilnehmer_innen ausgewählt wurden.

Gemeinsam mit den Motivatoren fragten wir auch nach Hindernissen, die mit dem Thema Wissenstransfer verbunden sind. Hierbei kristallisierten sich lediglich zwei Faktoren heraus: ,Nicht genügend zeitliche Ressourcen' (52,7\% Zustimmung) sowie, [Wissenstransfer] Ist für mein Vorankommen / meine Karriere nicht förderlich' (19,1\% Zustimmung). Ein signifikanter Unterschied mit kleiner Effektstärke ( $d_{\text {Co- }}$ hen $=-.41)$ konnte zwischen den verschiedenen wissenschaftlichen Disziplinen in Hinblick auf die verfügbaren zeitlichen Ressourcen festgestellt werden.

\begin{tabular}{|l|r|r|}
\hline Motivatoren & $\begin{array}{r}\text { Mittelwerts- } \\
\text { unterschied }\end{array}$ & $\begin{array}{r}\text { Effektstärke } \\
\left(d_{\text {Cohen }}\right)\end{array}$ \\
\hline Gesellschaftliche Aspekte & $-.251^{* * *}$ & .546 \\
\hline Um Wissen auch anwendbar zu machen & $.124^{*}$ & -.256 \\
\hline $\begin{array}{l}\text { Austausch mit Praktiker_innen/ } \\
\text { Firmen/Institutionen/etc. }\end{array}$ & $.198^{* *}$ & -.421 \\
\hline $\begin{array}{l}\text { Finanzielle Interessen (Projektförderungen, } \\
\text { Subventionen, etc.) }\end{array}$ & $.183^{* *}$ & -.427 \\
\hline $\begin{array}{l}\text { Vermarktung von gewonnenem Wissen (z.B. in } \\
\text { Form von Patenten o.ä.) }\end{array}$ & $.185^{* * *}$ \\
\hline $\begin{array}{l}\text { Tabelle 2: Ergebnisse der } t \text {-Tests für unabhängige Stichproben } \\
\text { (nichtsignifikante Ergebnisse sind in der Tabelle nicht angegeben) } \\
*=p<.05 ; * *=p<.01 ; * * *\end{array} \quad p<.001$ \\
\hline
\end{tabular}




\section{Einstellungen gegenüber dem Themenbereich Wissenstransfer}

Im letzten Teil der Studie präsentierten wir den Teilnehmer_innen eine Liste mit unterschiedlichen Aussagen zum Thema Wissenstransfer. Die einzelnen Items konnten auf einer 7-stufigen Likert-Skala von ,Stimme überhaupt nicht $z u^{\prime}$ bis ,Stimme voll und ganz zu' beurteilt werden.

Folgende Aussagen konnten den Antworten entnommen werden: Die Teilnehmer_innen bewerten Wissenstransfer als essenziellen und verpflichtenden Teilbereich der universitären Arbeit. Forschungsergebnisse werden durch Wissenstransfer nicht nur im Elfenbeinturm gehortet, sondern zu anderen gesellschaftlichen Bereichen disseminiert und somit auch zur Anwendung gebracht. Des Weiteren wurden neben der Rechtfertigung von Steuergeld auch der Auftrag zur Schaffung von öffentlichem Bewusstsein sowie der Beitrag zu gesellschaftlicher Verbesserung als wesentliche Aspekte angemerkt, die zu Wissenstransfer verpflichten. Trotz der moralischen Verbindlichkeiten sowie der vielseitigen Vorteile wurde erwähnt, dass das Engagement im Bereich Wissenstransfer innerhalb der Scientific Community nicht bzw. kaum wertgeschätzt werde und vor allem für ein Vorankommen in beruflicher Hinsicht nicht förderlich sei. Bezüglich des Themas Verwertung zeigten sich die Befragten eher skeptisch und befürworteten stattdessen die freie Verbreitung von universitär gewonnenen Forschungsergebnissen in die Gesellschaft. Letztlich wurde auch der aktuelle Usus bezüglich Kennzahlen und Benchmarking sehr kritisch bewertet. Die Teilnehmer_innen sprachen sich für eine Erweiterung der Kennzahlen aus und präzisierten, dass Leistungen in den Bereichen ,Science to Public' und ,Science to Professionals' ebenfalls in den Wissensbilanzen der Universitäten abgebildet werden sollten. 
Zur Untersuchung von möglichen Unterschieden zwischen GSKWissenschaftler_innen und Wissenschaftler_innen anderer Disziplinen wurde für jedes Item ein $t$-Test für unabhängige Stichproben berechnet. Die Ergebnisse zeigten signifikante Abweichungen in den Einstellungen hinsichtlich Verwertung, Bedeutung für die wissenschaftliche Karriere und Bedeutung der Tätigkeiten im Bereich ,Science to Public‘. Trotz der Signifikanz der Ergebnisse ist es wichtig hervorzuheben, dass beide Gruppen bei sämtlichen Items dennoch in die gleiche Richtung (Zustimmung bzw. Ablehnung) tendierten. Der Grad der Zustimmung/Ablehnung unterschied sich beinahe ausschließlich mit kleinen Effektstärken (Cohen, 1988). Lediglich zwei Items, die inhaltlich dem Thema Verwertung zuzuordnen sind, wiesen starke Mittelwertsunterschiede auf.

\section{Zusammenfassung}

Die Ergebnisse unserer Studie deuten darauf hin, dass Wissenschaftler_innen das Thema Wissenstransfer als bedeutsam und wichtig erachten. Trotz des ernstzunehmenden Stellenwerts scheint die Thematik jedoch innerhalb der Scientific Community kaum Wertschätzung zu erfahren. Diese Einstellung war von der überwiegenden Mehrheit der Befragten, unabhängig von der wissenschaftlichen Disziplin, erkennbar. In Übereinstimmung mit unseren Erwartungen konnte gezeigt werden, dass Wissenschaftler_innen ein sehr differenziertes Bild des Themenkomplexes haben. Dabei wurden jene Teilkomponenten, die als klassische Benchmarks etwa in Wissensbilanzen Eingang gefunden haben, deutlich seltener genannt als beispielsweise Themen aus den Bereichen ,Science to Public' und ,Science to Professionals'. Motivatoren konnten vor allem persönlichen und moralischen Motiven zugeordnet werden, wohingegen finanzielle Vorteile nur selten als motivierend bezeichnet wurden. Als Haupthindernisse kristallisierten 
sich die geringe Bedeutung für die berufliche Karriere sowie insbesondere ein Mangel an zeitlichen Ressourcen heraus.

Interessanterweise waren die Unterschiede im Antwortverhalten zwischen Forscher_innen der GSK-Wissenschaften und Forscher_innen anderer wissenschaftlicher Disziplinen deutlich geringer ausgeprägt als erwartet. Zwar konnten Unterschiede in Bezug auf die Teilbereiche des Themas Wissenstransfer sowie hinsichtlich der Einstellungen zum Thema festgestellt werden, doch bezogen sich die Differenzen lediglich auf den Grad der Zustimmung. Das Antwortverhalten der Befragten tendierte dennoch stets in die gleiche Richtung. 


\section{Literatur}

Agrawal, Ajay 2001. "University-to-Industry Knowledge Transfer: Literature Review and Unanswered Questions." International Journal of Management Reviews 3 (4): 285-302. doi:10.1111/1468-2370.00069.

Agrawal, Ajay and Rebecca Henderson. 2002. "Putting Patents in Context: Exploring Knowledge Transfer from MIT.” Management Science 48 (1): 44-60. doi: $10.1287 / \mathrm{mnsc}$.48.1.44.14279.

Caldera, Aida, and Olivier Debande. 2010. "Performance of Spanish Universities in Technology Transfer: An Empirical Analysis.” Research Policy 39 (9): 1160-73. doi:10.1016/j.respol.2010.05.016.

Cohen, Jacob. 1988. Statistical Power Analysis for the Behavioral Sciences. 2nd. ed. New Jersey: Lawrence Erlbaum Associates.

Cohen, Wesley M, Richard R Nelson, and John P Walsh. 2002. "Links and Impacts: The Influence of Public Research on Industrial R\&D.” Management Science 48 (1): 1-23. doi:10.1287/mnsc.48.1.1.14273.

European Commission. 2013. Knowledge Transfer Study 2010-2012. Brussels: European Union. doi:10.2777/31336.

Geuna, Aldo, and Alessandro Muscio. 2009. "The Governance of University Knowledge Transfer: A Critical Review of the Literature." Minerva 47 (1): 93-114. doi:10.1007/s11024009-9118-2.

Hayden, Markus C, Margarita K Petrova, and Daniel Wutti. 2018. "Direct Associations of the Terminology of Knowledge 
Transfer - Differences Between the Social Sciences and Humanities (SSH) and Other Scientific Disciplines." Trames: A Journal of the Humanities and Social Sciences 22 (3): 239-56. doi:10.3176/tr.2018.3.02.

Hayden, Markus C, Martin Weiß, Alice Pechriggl, and Daniel Wutti. 2018. "Insights Into University Knowledge Transfer in the Social Sciences and Humanities (SSH) and Other Scientific Disciplines - More Similarities Than Differences." Frontiers in Research Metrics and Analytics 3: 32. doi:10.3389/frma.2018.00032.

Lightowler, Claire, and Christine Knight. 2013. "Sustaining Knowledge Exchange and Research Impact in the Social Sciences and Humanities: Investing in Knowledge Broker Roles in UK Universities." Evidence and Policy 9 (3): 317-34. doi:10.1332/174426413X662644.

Molas-Gallart, Jordi, and Elena Castro-Martínez. 2007. "Ambiguity and Conflict in the Development of 'Third Mission' Indicators.” Research Evaluation 16 (4): 321-30. doi:10.3152/095820207X263592.

Olmos-Peñuela, Julia, Paul Benneworth, and Elena Castro-Martinez. 2014. "Are 'STEM from Mars and SSH from Venus'?:

Challenging Disciplinary Stereotypes of Research's Social Value." Science and Public Policy 41 (3): 384-400. doi:10.1093/scipol/sct071.

Olmos-Peñuela, Julia, Elena Castro-Martínez, and Pablo D’Este. 2014. "Knowledge Transfer Activities in Social Sciences and Humanities: Explaining the Interactions of Research Groups with Non-Academic Agents." Research Policy 43 (4): 696-706. doi:10.1016/j.respol.2013.12.004. 
Perkmann, Markus, Valentina Tartari, Maureen McKelvey, Erkko Autio, Anders Broström, Pablo D’Este, Riccardo Fini, et al. 2013. "Academic Engagement and Commercialisation: A Review of the Literature on University-Industry Relations." Research Policy 42 (2): 423-42. doi:10.1016/j.respol.2012.09.007.

Trencher, Gregory, Masaru Yarime, Kes B McCormick, Christopher N H Doll, and Steven B. Kraines. 2014. "Beyond the Third Mission: Exploring the Emerging University Function of CoCreation for Sustainability." Science and Public Policy 41 (2): 151-79. doi:10.1093/scipol/sct044.

Wutti, Daniel, and Markus Hayden. 2017. "Knowledge Transfer in the Social Sciences and Humanities (SSH) - Definition, Motivators, Obstacles, and Visions." Colloquium: New Philologies 2 (1): 87-101. doi:10.23963/cnp.2017.2.1.7. 